\title{
Symmetrical Review of Mobile App Icons and Their Effect on User Preferences $^{1}$
}

\author{
Oğuzhan Cinkara², Fatih Çağatay Baz ${ }^{2, *}$ \\ 2 OKU, Faculty of Economics and Administrative Sciences, Korkut Ata University, 80010 \\ Osmaniye, Turkey; fatihcagataybaz@osmaniye.edu.tr \\ * Correspondence: fatihcagataybaz@osmaniye.edu.tr
}

\begin{abstract}
With the widespread use of mobile phones, increasing use of mobile applications in different areas of life have gained a large place. Between the reasons of the preference of the rapid increasing number of apps in the application markets of the different platforms, the aesthetic appereance of the icons that apps have perhaps the most important one. In this study, the visual symmetrical side of the icons of the most downloaded apss which are developed for preparing to Public Servant Exam in Turkey is emphasized. Two different types of data are obtained with working on the icons with image processing technique by using Mathworks Matlab program and survey method which is applied on Korkut Ata University students. By comparing the obtained data with the binary logistic regression method, it was determined that the visual symmetry in the apps' icons partially contributed to the aesthetic appreciation.
\end{abstract}

Keywords: symmetry, symmetry detection techniques, image processing, matlab.

\footnotetext{
${ }^{1}$ This article is extracted from master thesis of Oğuzhan Cinkara's " Examining User Preferences in Terms of Visual Design in Mobile Education Applications",
} 


\section{Introduction}

Advances in science and technology have become a necessity for societies to catch up with the needs of the age. It is also seen that the use of technology in education has become widespread in recent years. Today's understanding of education has gone beyond the classical teacher-student-board triangle. Schooling can be done with more than one method and technique.

Mobile education is a method of reaching the desired information with various devices at any time and from anywhere. In other words, it's a learning activities without being tied to a class or lecturer [1].

Mobile application is a program designed for users to perform certain operations on mobile devices such as tablet phones and watches [2, 3].

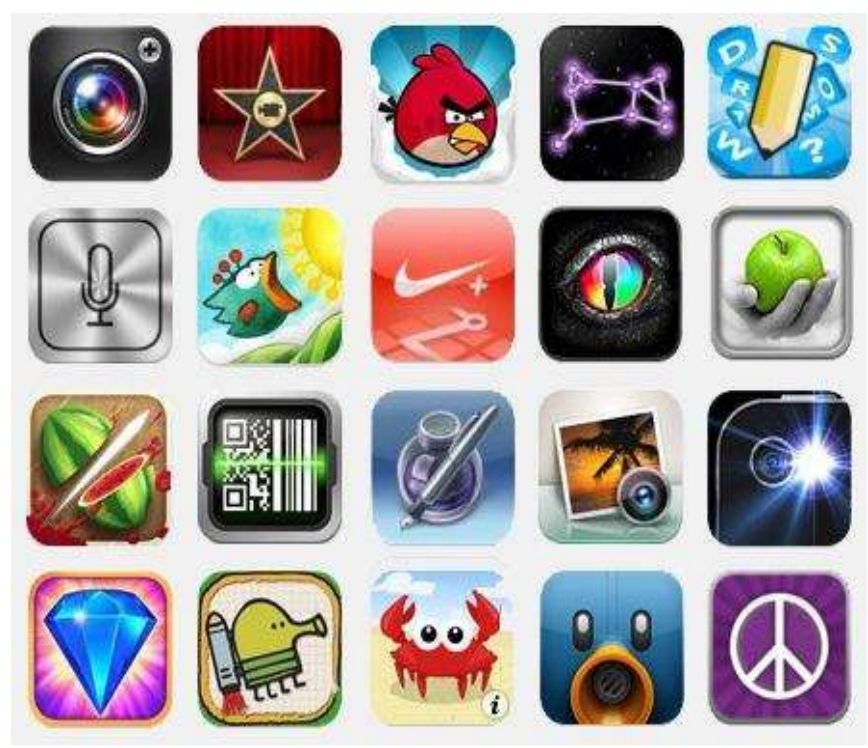

Fig 1. Application Icons

The Word of icon comes from the Greek word "eikon" and it means picture. It is also defined as a religious totem in the dictionary. Icons are derived from signs that express some concepts in a sense or some way for someone [4]. 


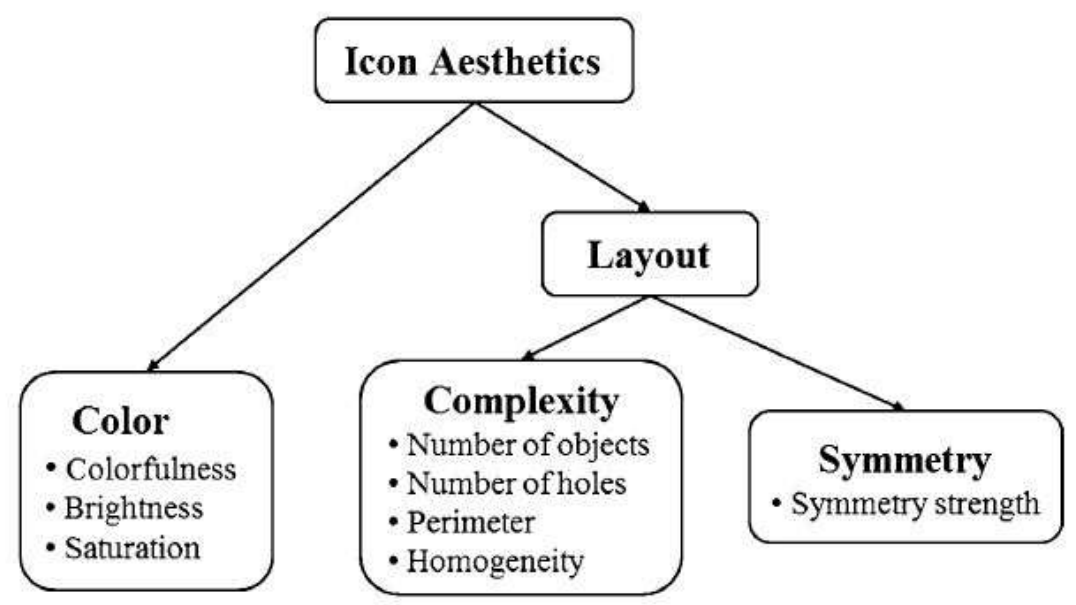

Fig 2. Icon Aesthetics [5]

Lavie and Tractinsky (2004) [6] and Moshagen and Thielsch (2010) [7] stated that classical aesthetics originated from simplicity and consisted of visual configurations such as regularity of openness, homogeneity, grouping, balance and symmetry.

Symmetry is an internal phenomenon that occurs both naturally and man-made in the world around us. It is attractive as a clue directing both aesthetic and visual attention. Symmetry is naturally found in the structure of our world in many different scales and in many different forms. It is evident in the shape and appearance of many natural and manmade scenes. Humans have an innate ability to perceive symmetries in objects and images [9-11].

The two most common types of symmetry are rotational and mirror (reflection) symmetry. Reflection, rotation and translation symmetries have been the subject of numerous detection methods in recent years. Of the three, reflection symmetry is the most common as in the real world $[14,15]$.

In this study;

-We investigated whether the apps' preference by the people increases as the symmetry of the icons of the apps' increases.

-We focused on reflection type of symmetry and used Mathworks MATLAB 2015 software for image processing.

-We restricted our study with 10 most downloaded education app icons about the Public Personnel Election Exam (aka. Kpss) held annually for public employment in Turkey.

-We did a survey which based on giving a credit point between 1 to 5 for every app's icon, among the 172 students of Korkut Ata University Economics and Administrative Sciences Faculty.

-We compared the symmetry values of icons obtained from matlab software which is between 0-1 with the survey results of the icons between the 1-5 by the binary logistic regression method. 


\section{Related Works}

There are many studies on the symmetrical and aesthetic evaluations of pictures with image processing method.

Wang and Li (2017) [13] proposed a multi-step aesthetic analysis model to investigate the effects of the aesthetics of app icons on the number of downloads of apps. In their model they evaluated the effects of the aesthetic design on the number of downloads with the various formulas, which developed on the features like app icon's complexity, symmetry and simplicity etc.

Cornelius ve Loy (2006) [8] worked on a symmetry detection algorithm which combines the SIFT descriptor with several different feature detection methods such as hessian-affine and Harris affine detectors, an intensity extrema-based detector (IBR) and an edge based region detector (EBR).

Shi et al. (2018) [12] used root-mean-squared-error (RMSE) for detecting the symmetry in the shapes of the leaves by splitting their images into parts in the matlab.

Widynski et al. (2014) [14] 's approach is used Ribbon Detection method to find the regional symmetries by extracting the linear lines of the objects in the image to draw the lines of the image itself. Their approach also proposes a sequential Monte Carlo method, a particle filter application, to extract part of the structure at once.

\section{Methodology}

Icon images of the first most preferred 10 kpss exam application, downloaded from the Google Play Store in .jpeg, .png format on December 12, 2018 (ranking of the applications may have changed). The images turned into pixel format in Mathworks Matlab 2015 software. Since method we proposed in this study is based on the detection of white pixels, we turned every images of the icons into black and white pixels with one of the Matlab's edge detection method called "canny". Our method works around the axes that are considered to pass right through the middle of the picture in horizontal and vertical directions.

The applications whose icons we are worked on are named as "Application $1, \ldots, 10$ " according to the number of their downloads.

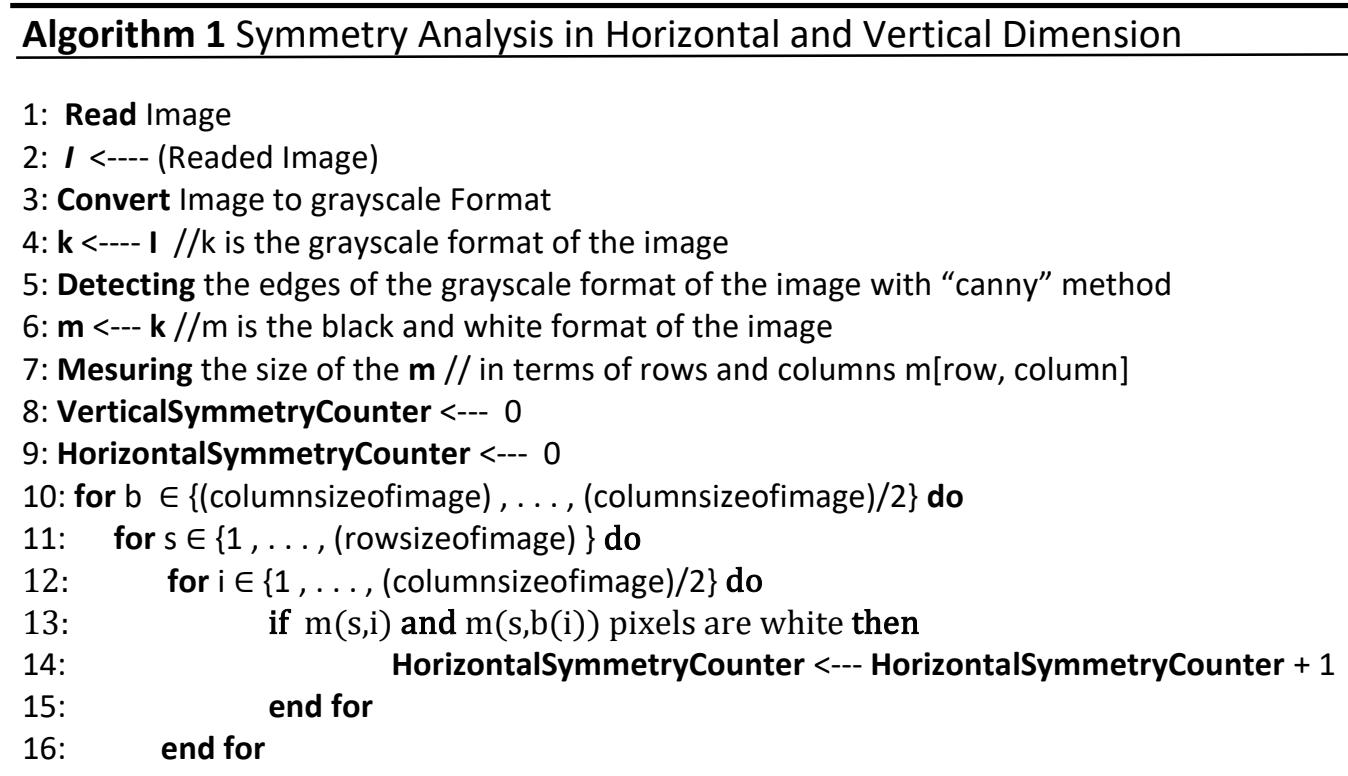


17: end for

18: end for

19: for $b \in\{($ rowsizeofimage),$\ldots$, (rowsizeofimage $) / 2\}$ do

20: for $s \in\{1, \ldots$, (columnsizeofimage) $\}$ do

21: $\quad$ for $i \in\{1, \ldots$, (rowsizeofimage) $/ 2\}$ do

22: $\quad$ if $\mathrm{m}(\mathrm{i}, \mathrm{s})$ and $\mathrm{m}(\mathrm{b}(\mathrm{i}), \mathrm{s})$ pixels are white then

23: VerticalSymmetryCounter <--- VerticalSymmetryCounter +1

24: $\quad$ end for

25: end for

26: end for

27: end for

28: TotalWhitePixelCounter <--- 0

29: for $s \in\{1, \ldots$, (columnsizeofimage) $\}$ do

21: $\quad$ for $i \in\{1, \ldots$, (rowsizeofimage) $\}$ do

22: $\quad$ if $\mathrm{m}(\mathrm{s}, \mathrm{i})$ pixel is white then

23: $\quad$ TotalWhitePixelCounter $<---$ TotalWhitePixelCounter +1

24: end for

25: end for

26: VerticalSymmetryofthelmage <--- VerticalSymmetryCounter/ TotalWhitePixelCounter

27: HorizontalSymmetryofthelmage <--- HorizontalSymmetryCounter/ TotalWhitePixelCounter

28: TotalSymmetrofthelmage<--- (VerticalSymmetryofthelmage + HorizontalSymmetryofthelmage ) /2

\section{Matlab Codes}

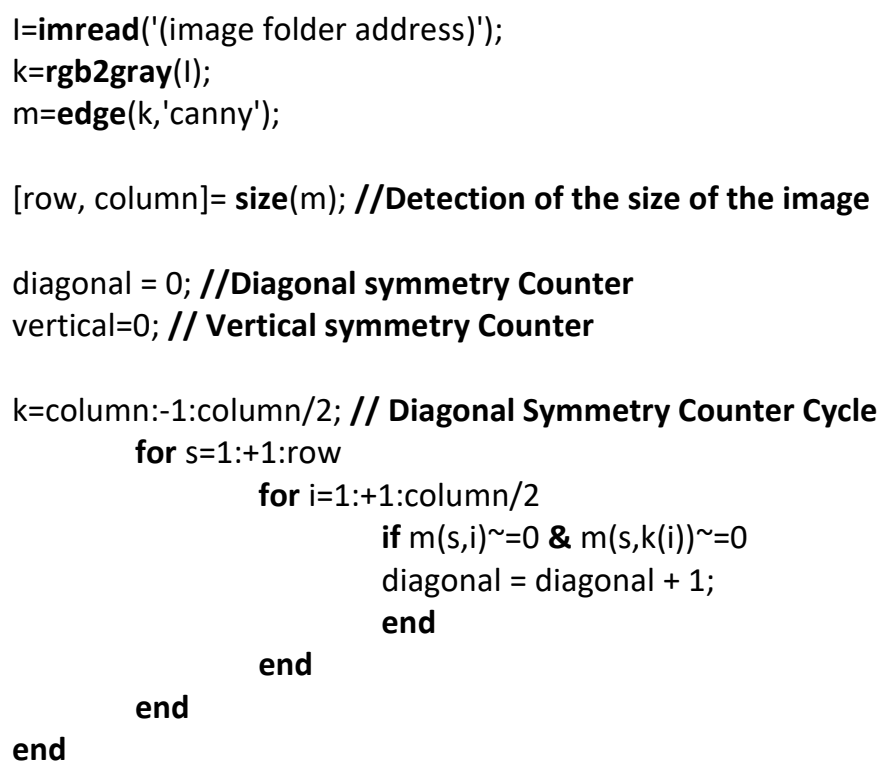

figure,imshow $(m)$ //Drawing Diagonal Symmetry Lines

hold on

for $s=1:+1$ :row

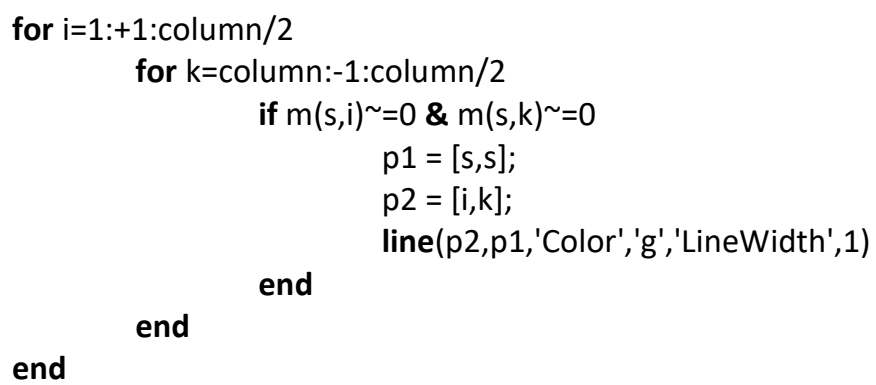




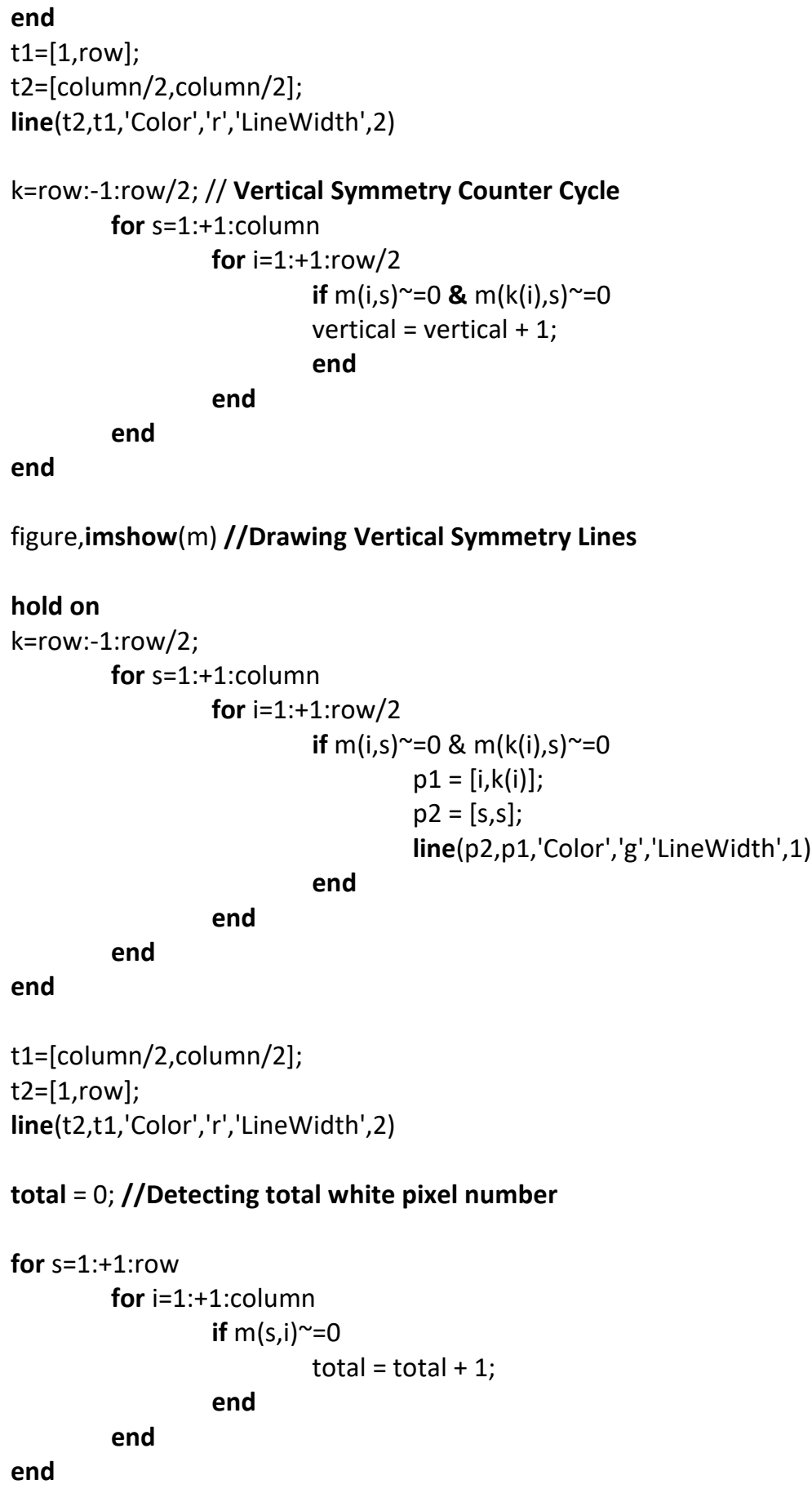

In fig 1 and 2, our algorithm for detecting symmetry has been described by showing pixels of an images as squares. For detecting white pixels which have same distance to the middle row and column in horizontal and vertical dimensions, our algorithm starts searching from opposite sides which are showed in the figs at the same time. 


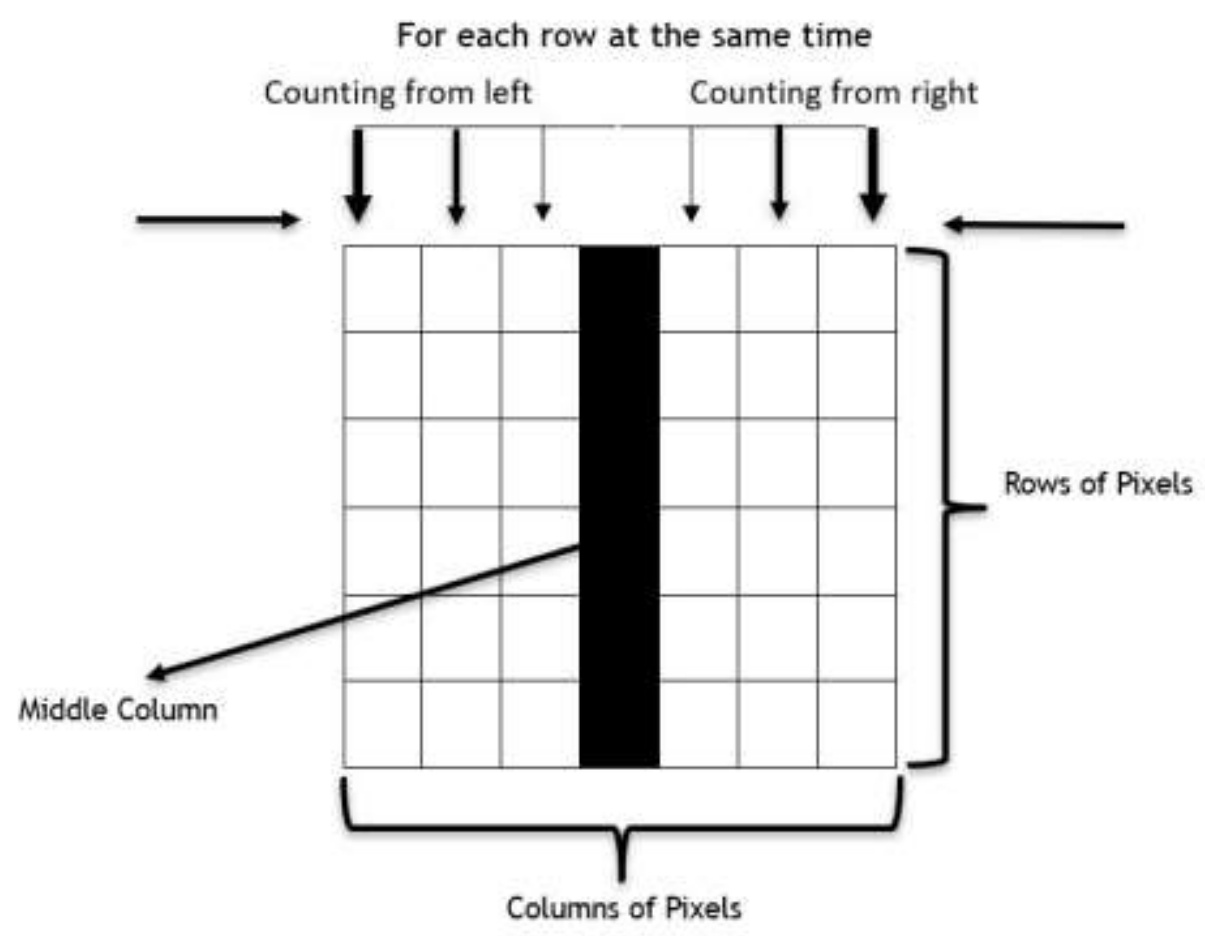

Fig 3. Measuring the horizontal symmetry

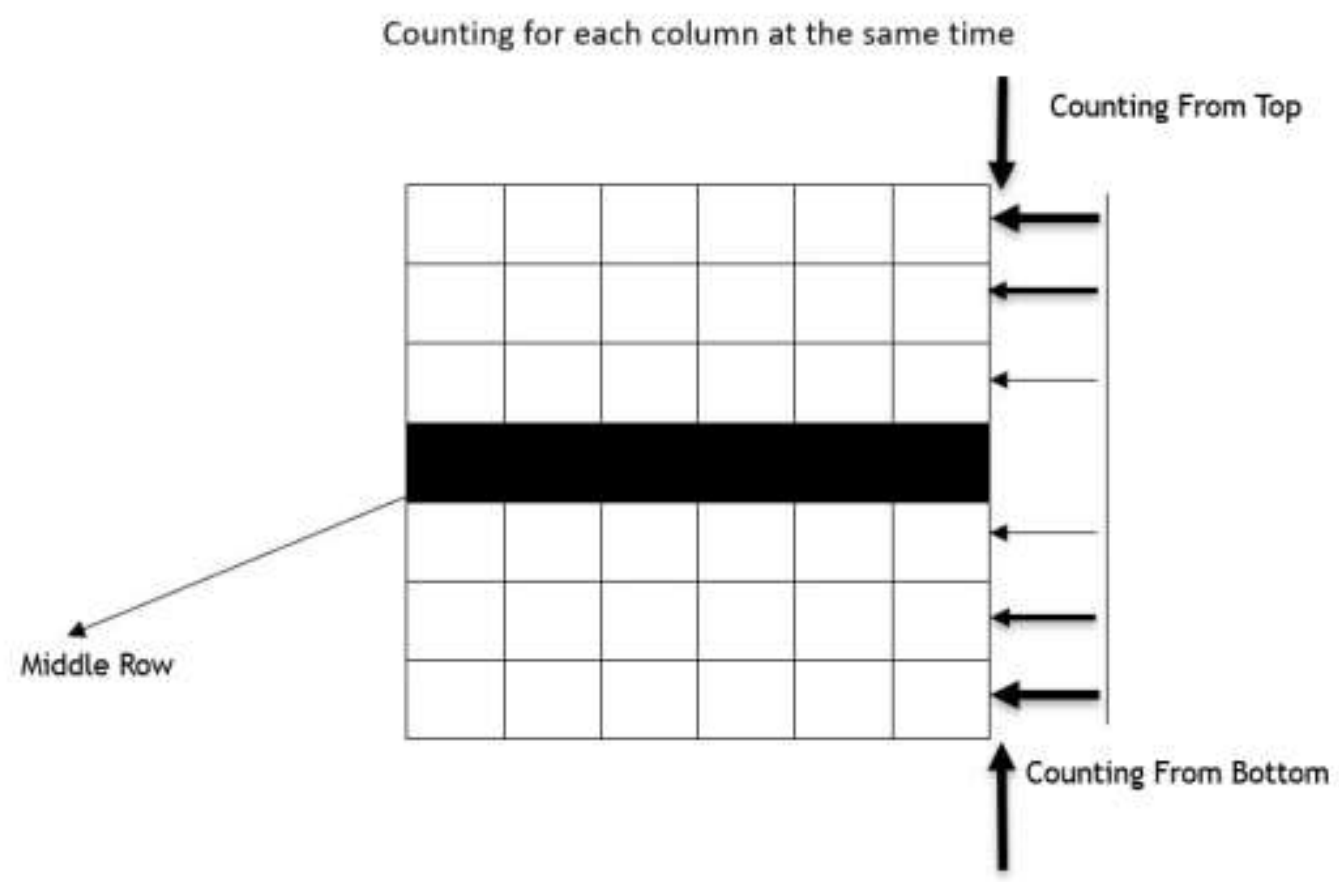

Fig 4. Measuring the vertical symmetry 
In Figure 5, application of our method's firs chapter about turning image into blackwhite pixel form with matlab's "canny" method is shown on one of the app's icon image we examined in this study.

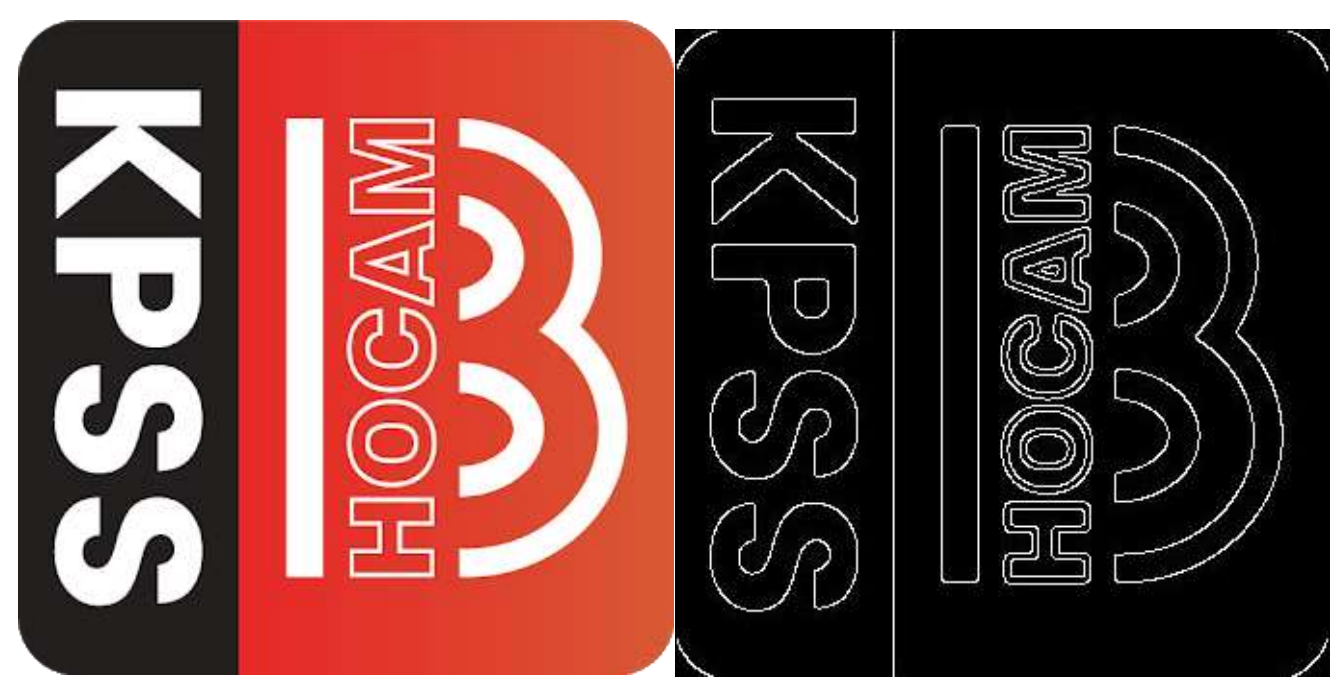

Fig 5. Turning Icon Image into Black and White Pixel Form

In Figure 6, the red straight vertical line drawn in the matlab program, which we accept as the horizontal symmetry axis passing through the center of the icon is shown.

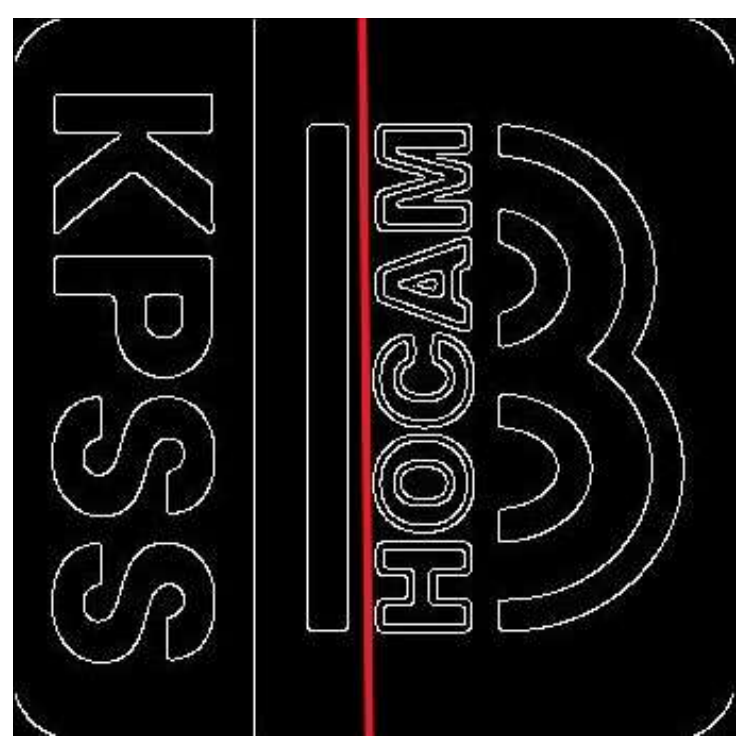

Fig 6. Drawing the horizontal symmetry axe

In Figure 7, white pixels that are equidistant from the axis in the same plane are shown by matching the green lines from the parts where this axis is divided equally into two. 


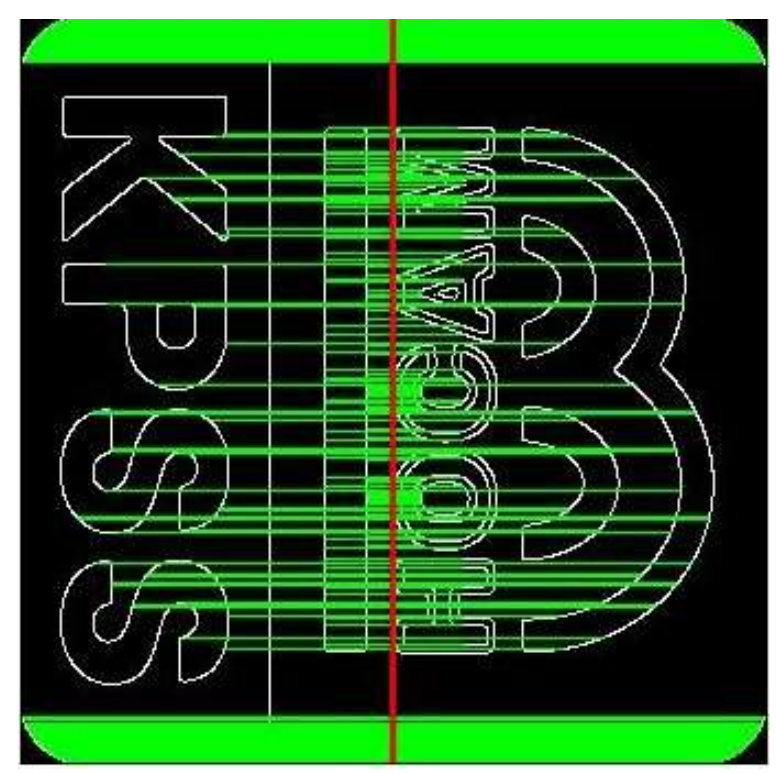

Fig 7. White Pixels Equal Distance to the Symmetry Axis on the Horizontal Axis

The horizontal symmetry ratio which is between 0 and 1 , was determined with dividing the number of matching white pixels by the total number of white pixels in the picture.

The application of the same process on the horizontal axis is shown in Figure 8 and 9.

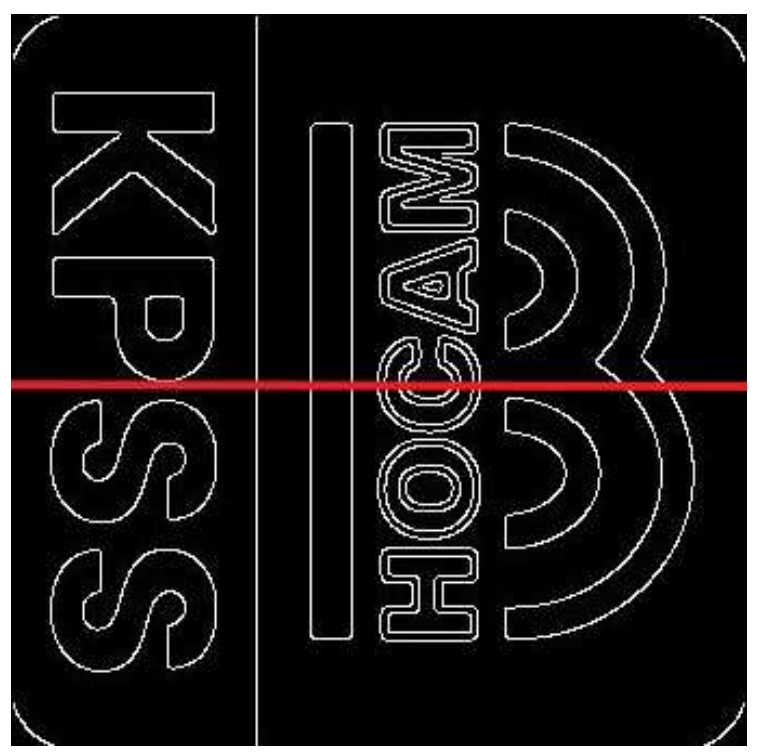

Fig 8. Drawing the vertical symmetry axe 


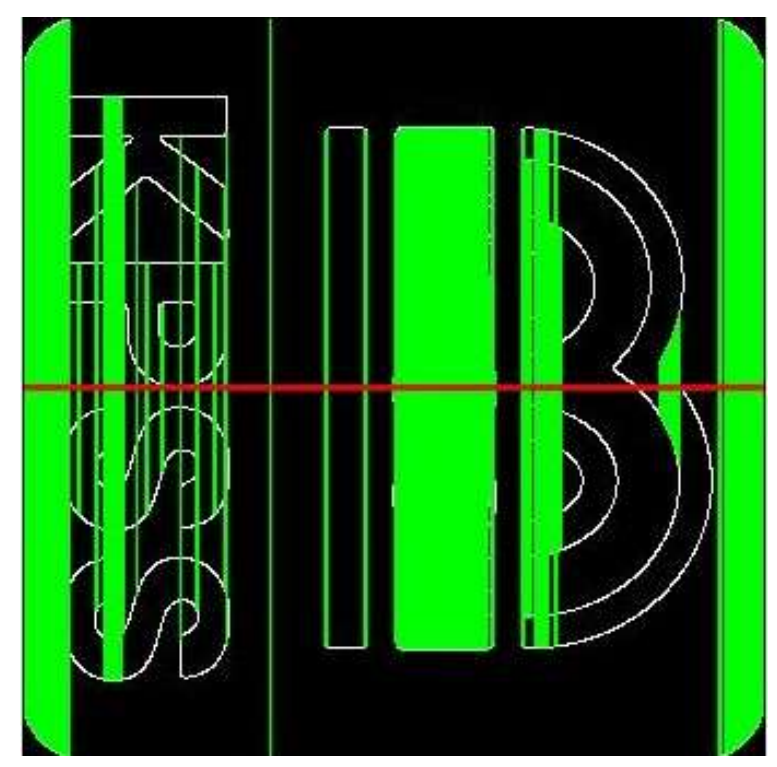

Fig 9. White Pixels Equal Distance to Symmetry Axis on Vertical Axis

By taking the average of the horizontal symmetry and vertical symmetry ratios, the general symmetry ratio of the picture was found between $0-1$.

\section{Survey}

A survey which is shown in the figure 10 has been applied to the students of the Korkut Ata University Faculty of Economics and Administrative Sciences. Participants were asked to score the app icons on which we are working on a scale numbered from 1 (Very Bad) to 5 (Very Good).

By dividing the total scores of the icons from the survey by the number of participants, an average survey score between 1 and 5 was obtained for each icon.

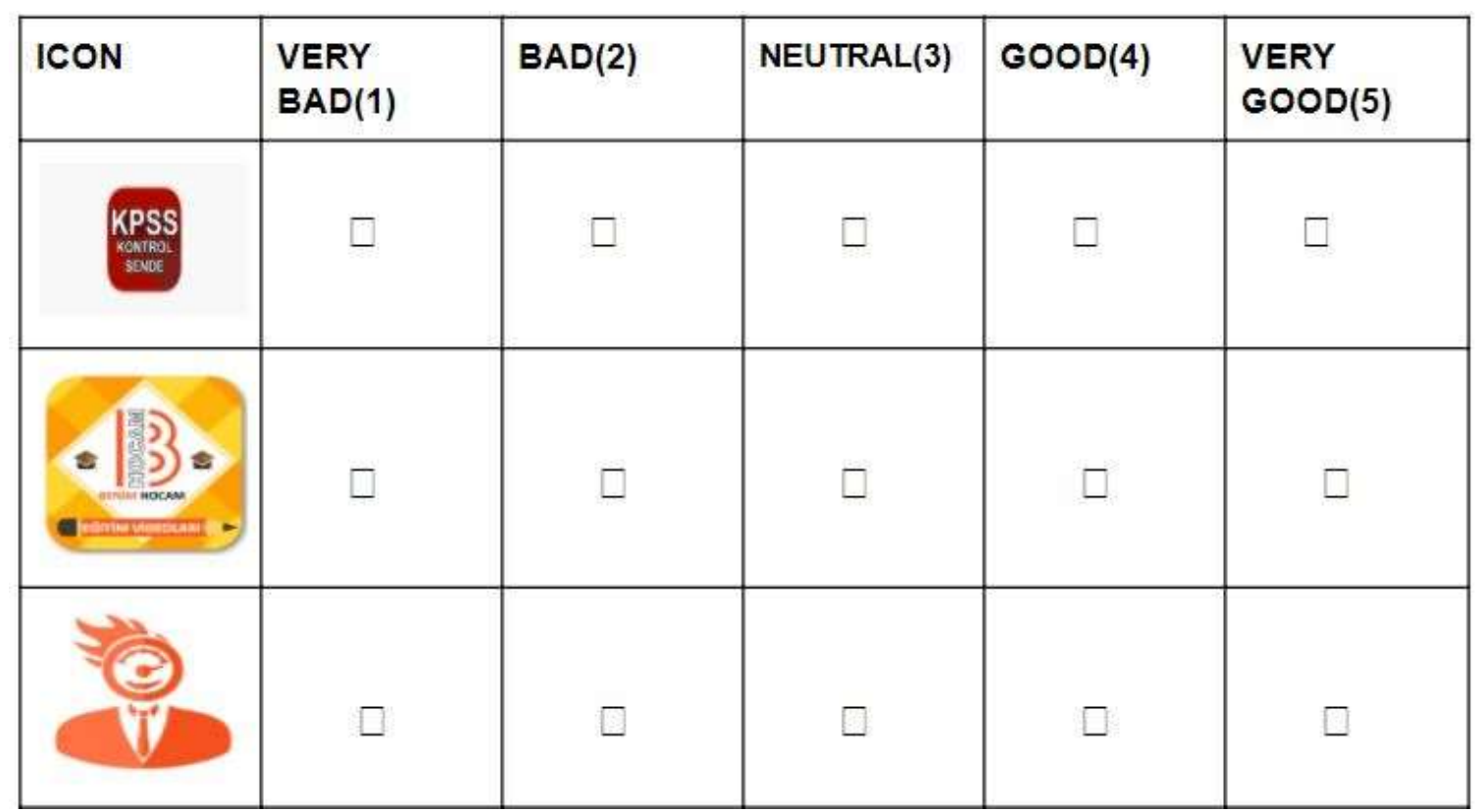

Fig 10. Rating the Icons 


\section{Evaluation of the Results}

Icons with a general average or above of the values obtained from the survey are classified as "AESTHETIC" and icons that are below the general average are classified as "NOT AESTHETIC". Survey values accepted as a dependent variable in the binary logistic regression.

Matlab values were divided into two groups as the general average or above and those below the general average. Matlab values accepted as an independent variable.

In accordance with the classifications, sensitivity analysis was made over the "odds" values extracted from the error matrix by placing the icon numbers that meet the appropriate conditions in the $2 \times 2$ error matrix in Figure 11.

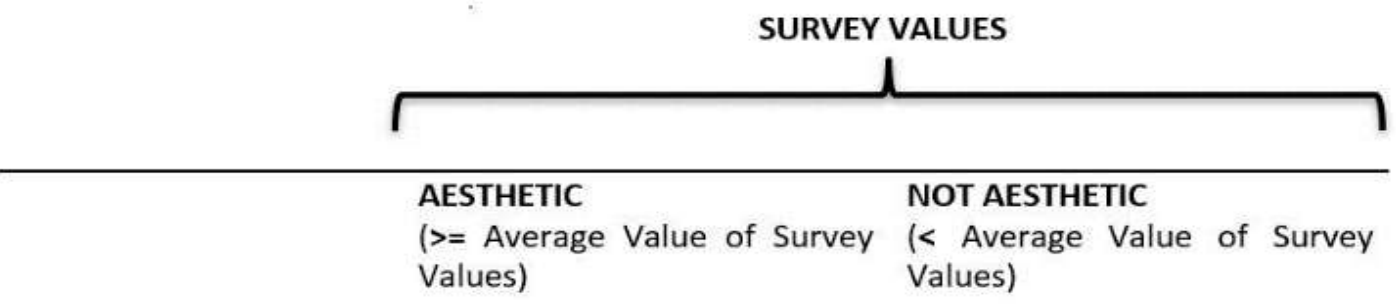

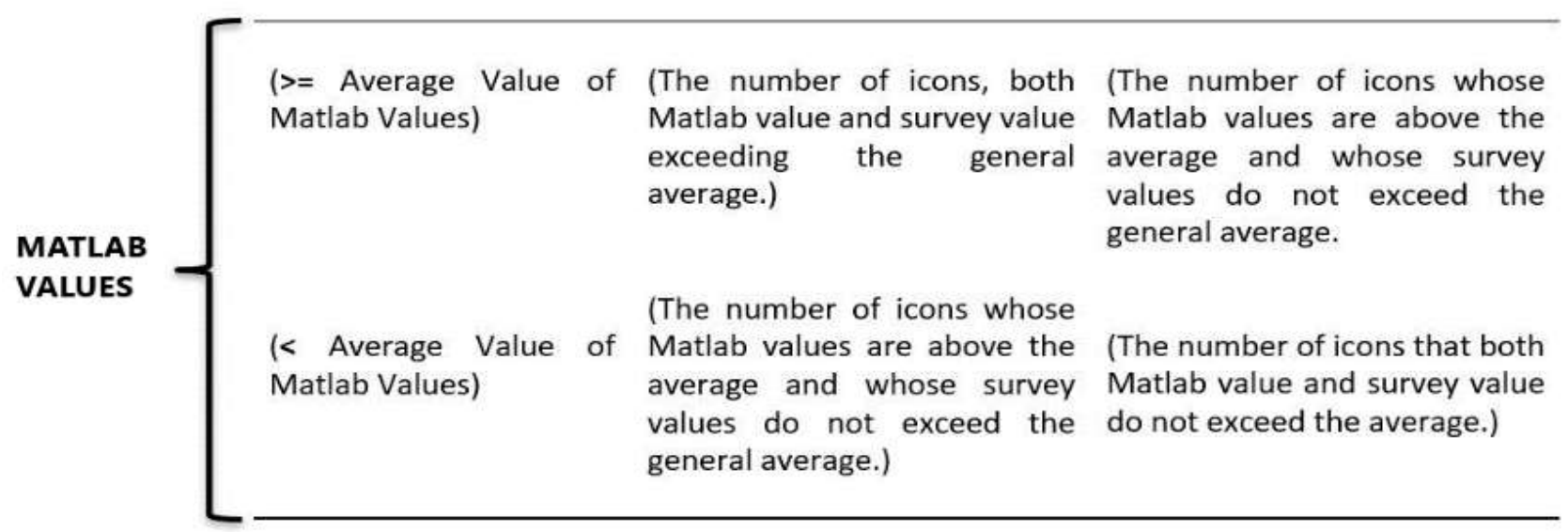

Fig 11. Confusion Matrix (2x2) 


\section{Results}

\subsection{Results of Application Number 1}

App Name: Kpss Kontrol Sende

Number of Download: 1 Million +

Google Play Point: 4,6

Horizontal Symmetry Value: 0.11

Vertical Symmetry Value: 0.07

Total Symmetry Value: 0.09

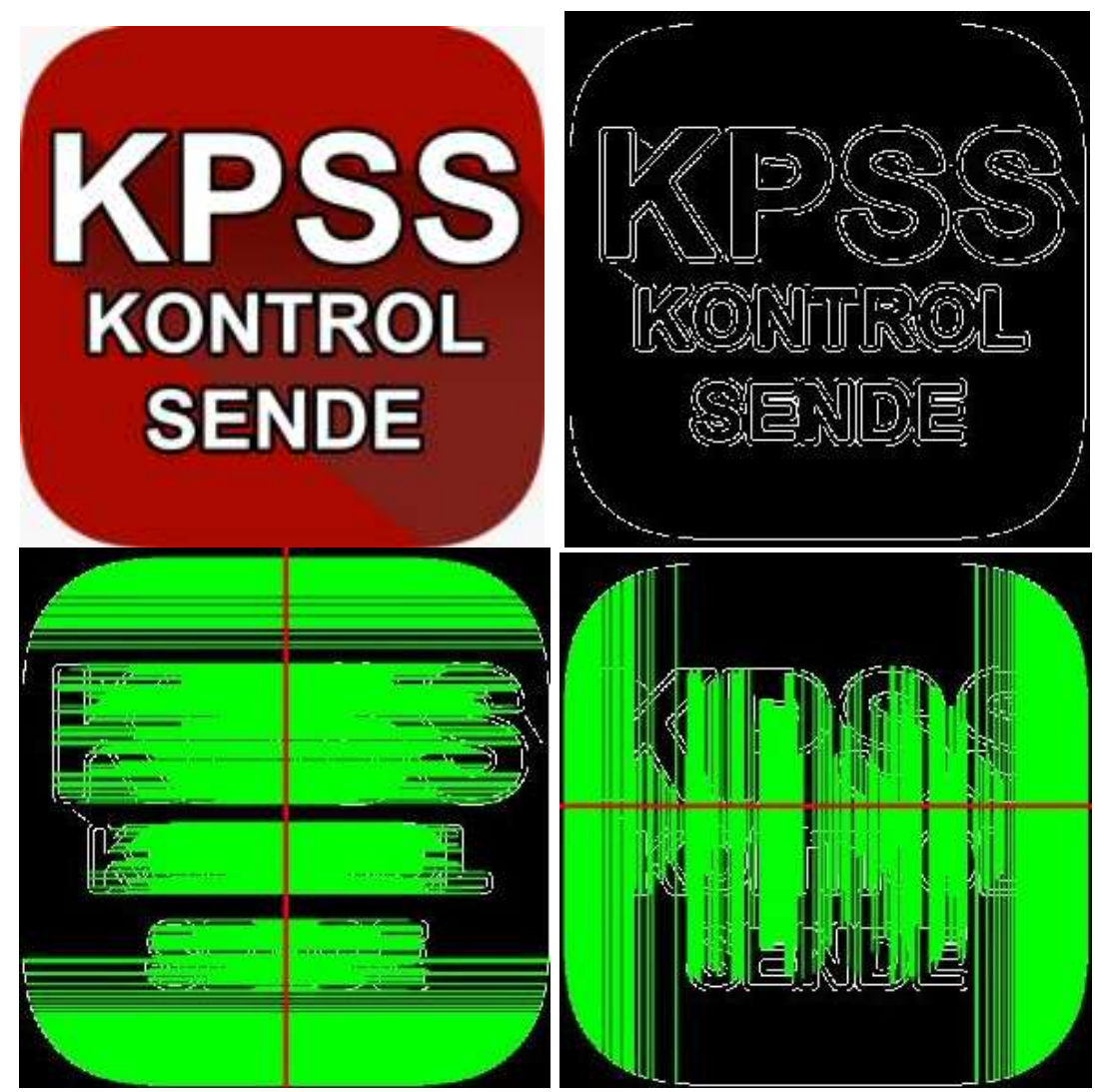

Fig 12. Image Processing Results of Application 1

Figure 12 shows the image processing steps of the application 1 icon. Density was observed in the number of matching white pixels in the horizontal plane and the horizontal symmetry value was found to be 0.11 . The density of the matching white pixels on the vertical axis was observed occasionally and the vertical symmetry value was determined as 0.07 . The total symmetry value was determined as 0.09 . 


\subsection{Results of Application Number 2}

App Name: Benim Hocam Mobil Number of Download: $100.000+$ Google Play Point: 4,8 Horizontal Symmetry Value: 0.18 Vertical Symmetry Value: 0.06 Total Symmetry Value:0.12

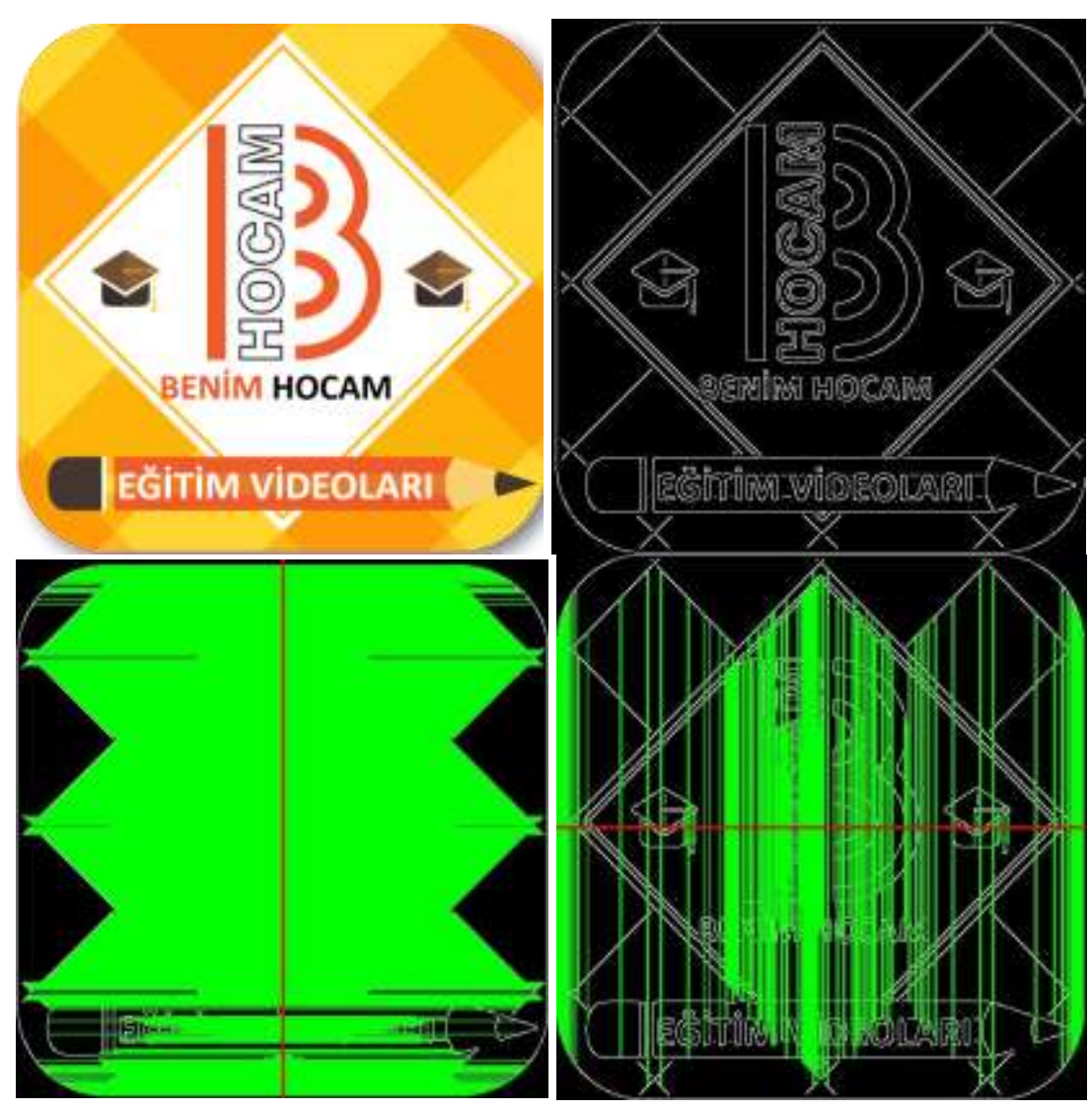

Fig 13. Image Processing Results of Application 2

Figure 13 shows the image processing steps of the application 2 icon. The density was observed in the number of matching white pixels in the horizontal plane and the horizontal symmetry value was determined as 0.18 . The same density was not observed in the number of matching white pixels on the vertical axis and the vertical symmetry value was determined as 0.06 . The total symmetry value is determined as 0.12 . 


\subsection{Results of Application Number 3}

App Name: KPSS'ye Hazırlanıyorum

Number of Download: $100.000+$

Google Play Point: 4,5

Horizontal Symmetry Value: 0.03

Vertical Symmetry Value: 0.03

Total Symmetry Value: 0.03

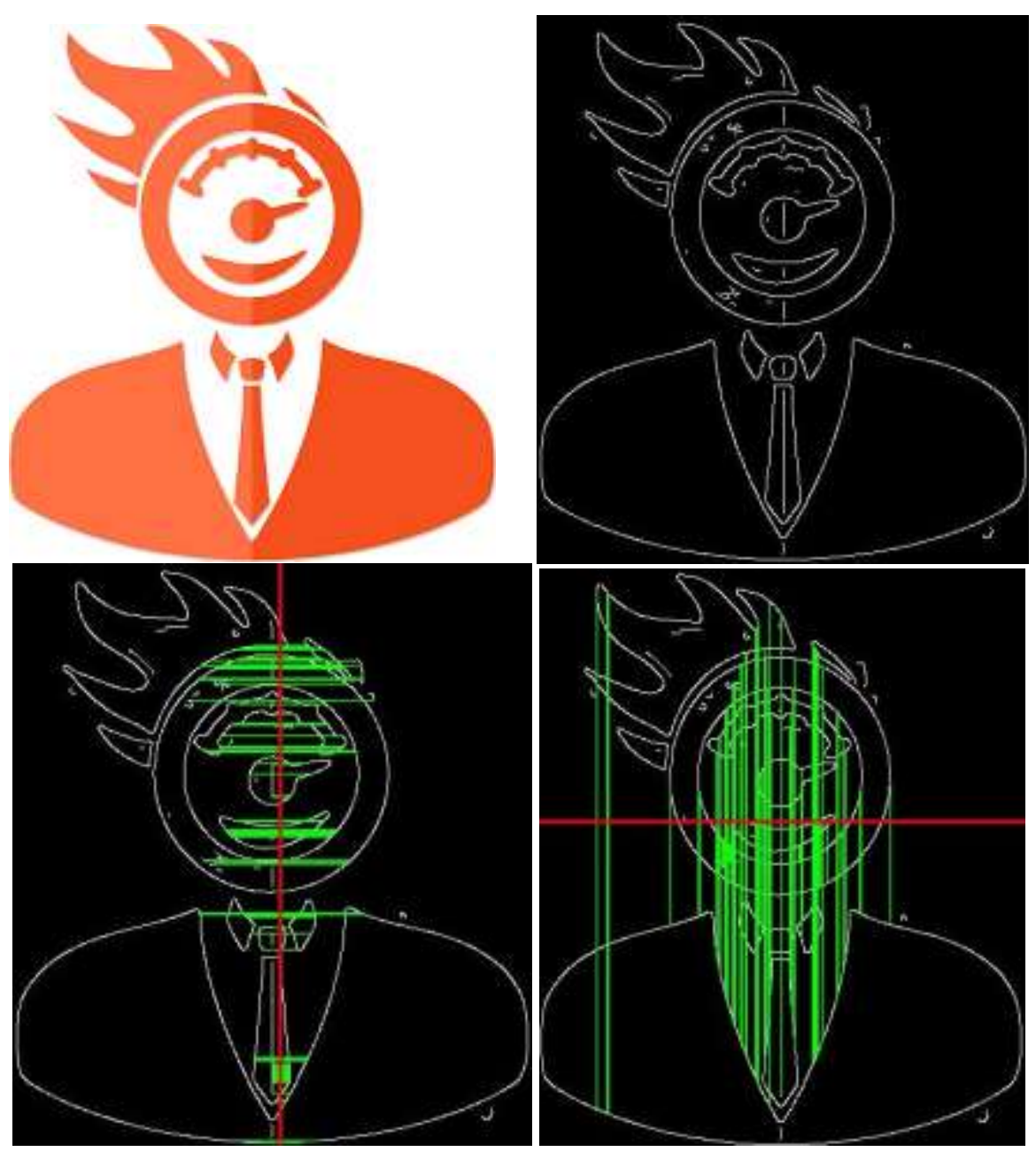

Fig 14. Image Processing Results of Application 3

Figure 14 shows the image processing steps of the application 3 icon. Low density was observed in the number of matching white pixels in the horizontal plane and the horizontal symmetry value was determined as 0.03 . The same low intensity was observed in the number of matching white pixels on the vertical axis and the vertical symmetry value was determined as 0.03 . The total symmetry value was determined as 0.03 . 


\subsection{Results of Application Number 4}

App Name: KPSS Cebimde 2019

Number of Download: $100.000+$

Google Play Point: 4,5

Horizontal Symmetry Value: 0.05

Vertical Symmetry Value: 0.04

Total Symmetry Value: 0.045

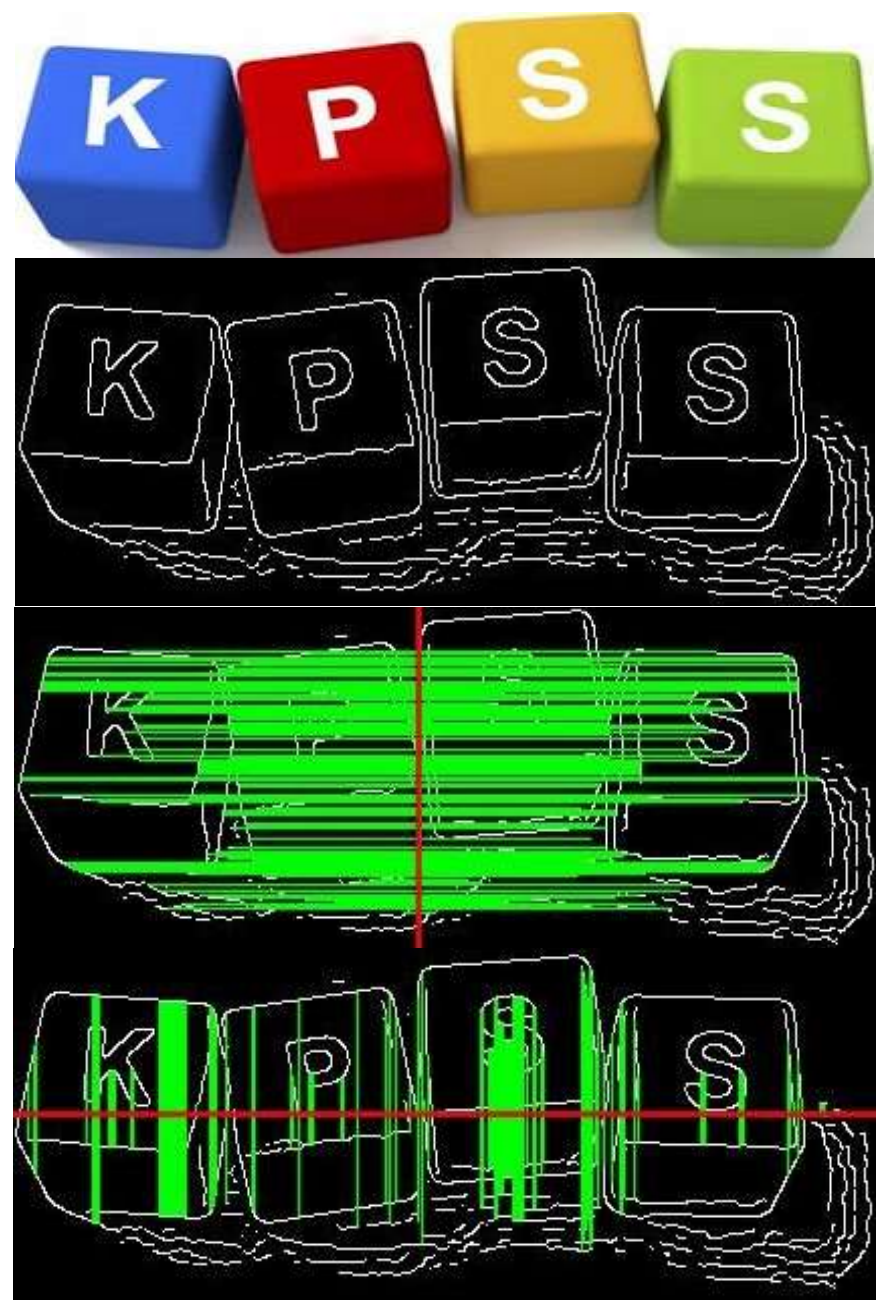

Fig 15. Image Processing Results of Application 4

Figure 15 shows the image processing steps of the application 4 icon. Density was observed in the number of matching white pixels in the horizontal plane and the horizontal symmetry value was determined as 0.05 . Low density was observed in the number of matching white pixels on the vertical axis and the vertical symmetry value was determined as 0.04 . The total symmetry value was determined as 0.045 . 


\subsection{Results of Application Number 5}

App Name: KPSS Cafe

Number of Download: $100.000+$

Google Play Point: 4,0

Horizontal Symmetry Value: 0.04

Vertical Symmetry Value: 0.02

Total Symmetry Value: 0.03
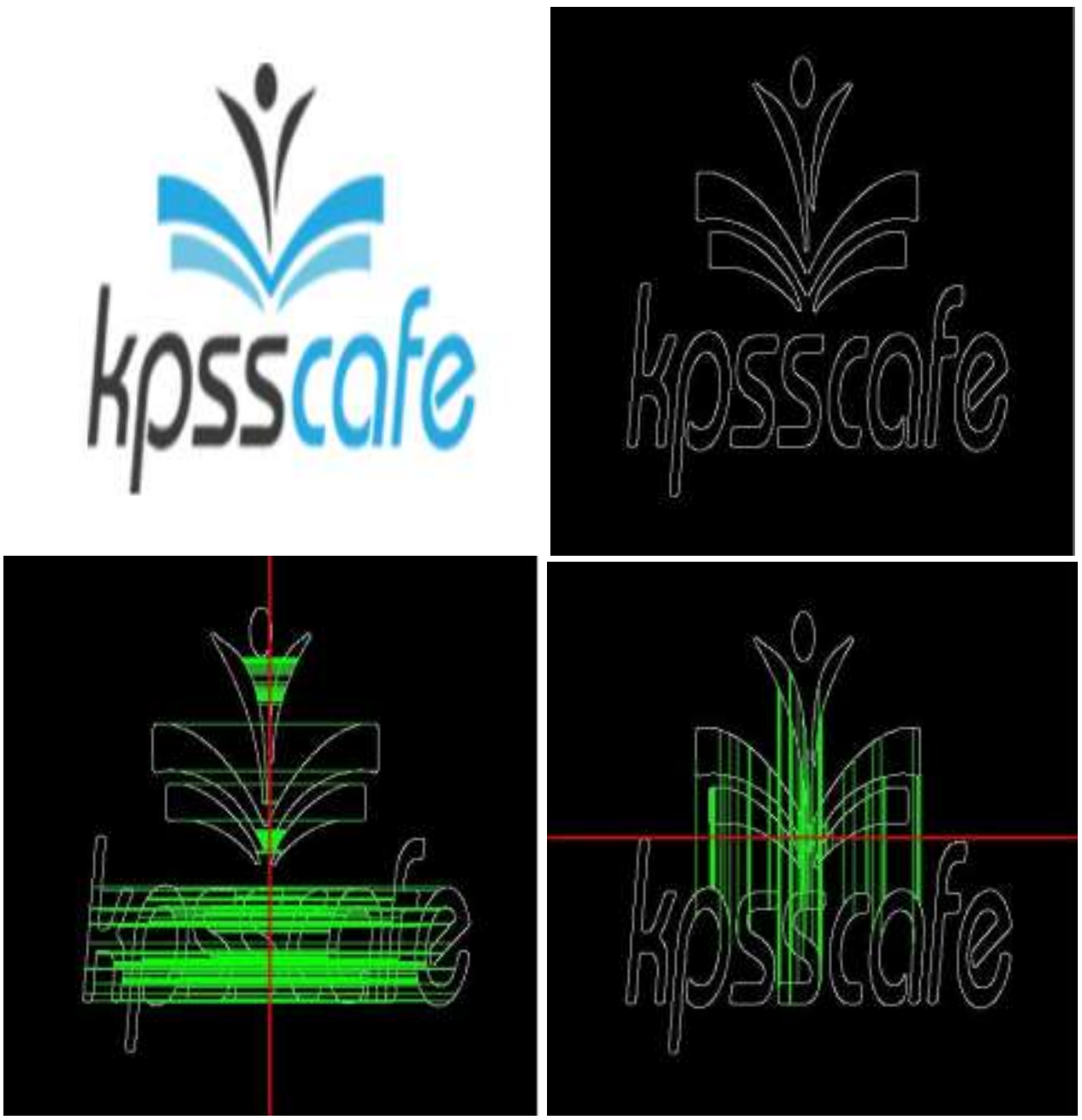

Fig 16. Image Processing Results of Application 5

Figure 16 shows the image processing steps of the application 5 icon. Low density was observed in the number of matching white pixels in the horizontal plane and the horizontal symmetry value was determined as 0.04 . Low density was observed in the number of matching white pixels on the vertical axis and the vertical symmetry value was determined as 0.02 . The total symmetry value was determined as 0.03 . 


\subsection{Results of Application Number 6}

App Name: KPSS Milyoner

Number of Download: $50.000+$

Google Play Point: 4,1

Horizontal Symmetry Value: 0.22

Vertical Symmetry Value: 0.12

Total Symmetry Value: 0.17

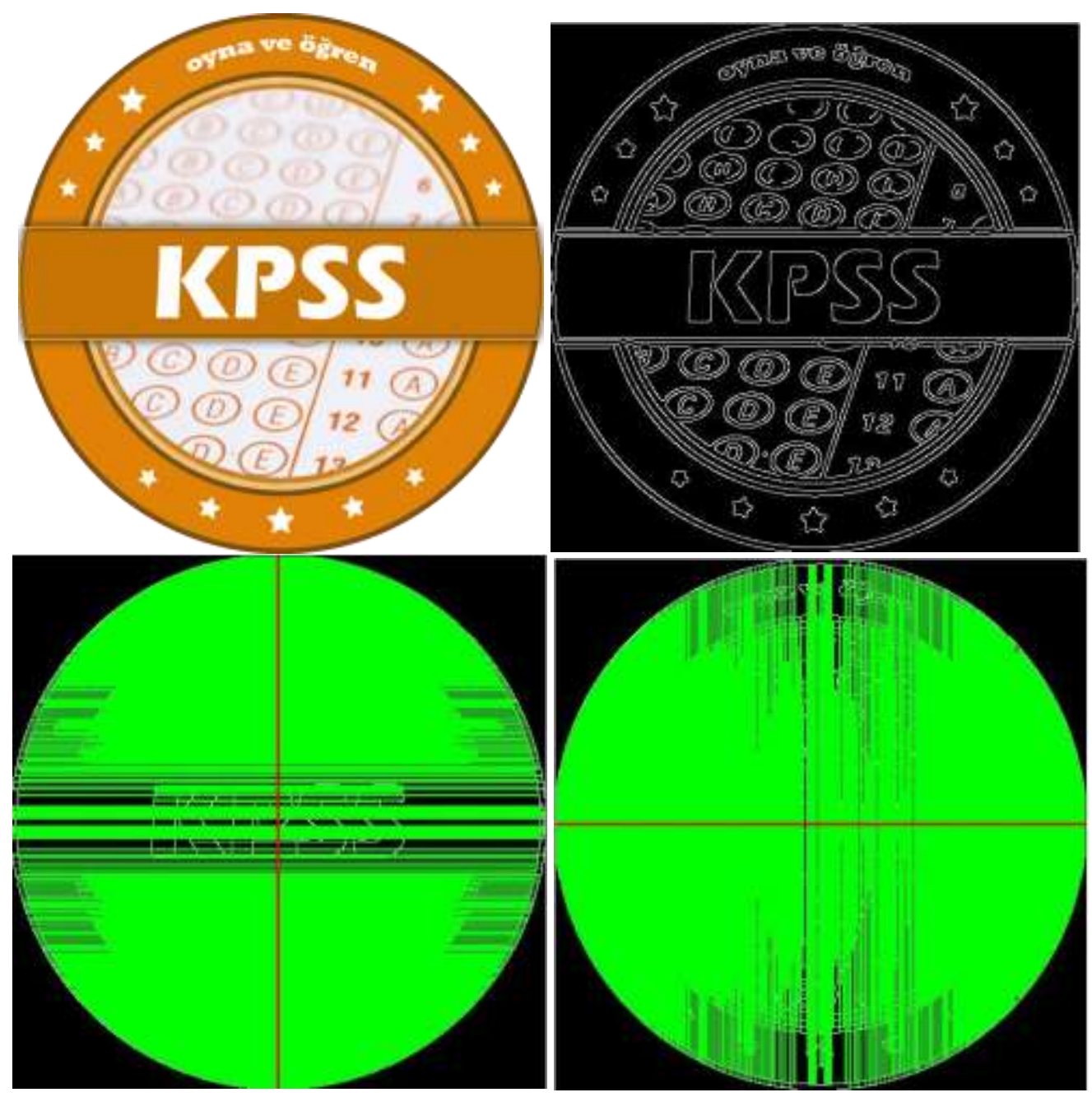

Fig 17. Image Processing Results of Application 6

Figure 17 shows the image processing steps of the application 6 icon. Excessive density was observed in the number of matching white pixels in the horizontal plane and the horizontal symmetry value was determined to be 0.22 . Excessive density was observed in the number of matching white pixels on the vertical axis and the vertical symmetry value was determined as 0.12 . The total symmetry value was determined as 0.17 . 


\subsection{Results of Application Number 7}

App Name: KPSS Aşkım 2019

Number of Download: $50.000+$

Google Play Point: 4,8

Horizontal Symmetry Value: 0.06

Vertical Symmetry Value: 0.03

Total Symmetry Value: 0.045
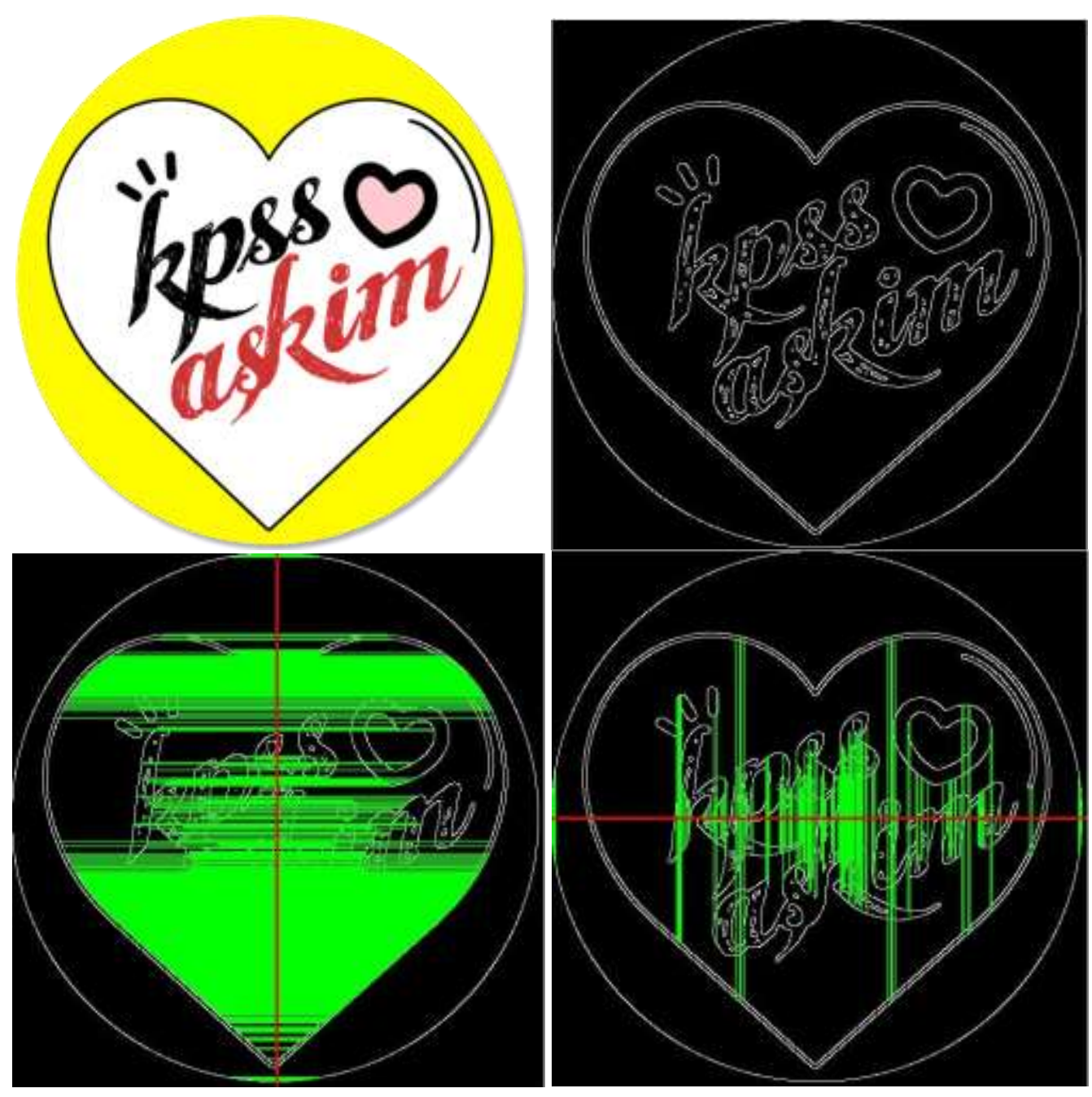

Fig 18. Image Processing Results of Application 7

Figure 18 shows the image processing steps of the application 7 icon. Partial density was observed in the number of matching white pixels in the horizontal plane and the horizontal symmetry value was determined as 0.06 . Sparse density was observed in the number of matching white pixels on the vertical axis and the vertical symmetry value was determined as 0.03 . The total symmetry value was determined as 0.045 . 


\subsection{Results of Application Number 8}

App Name: Kpss Son 10 Yıl Çıkmış Sorular 2006-2017

Number of Download: 50.000+

Google Play Point: 4,0

Horizontal Symmetry Value: 0.09

Vertical Symmetry Value: 0.05

Total Symmetry Value: 0.07

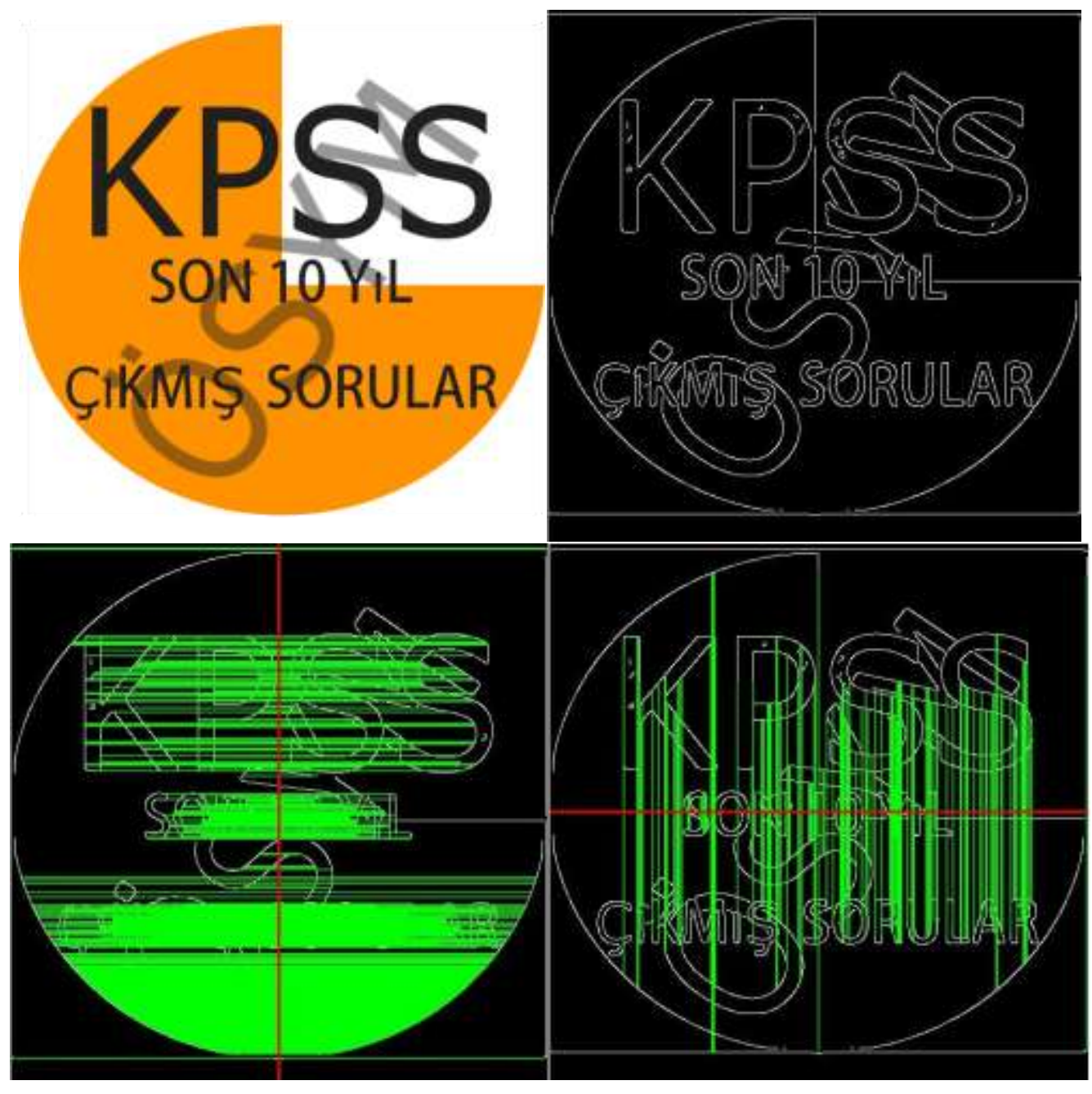

Fig 19. Image Processing Results of Application 8

In Figure 19 image processing steps of the application 8 icon are shown. Partial density was observed in the number of matching white pixels in the horizontal plane and the horizontal symmetry value was determined as 0.09 . Sparse density was observed in the number of matching white pixels on the vertical axis and the vertical symmetry value was determined as 0.05 . The total symmetry value was determined as 0.07 . 


\subsection{Results of Application Number 9}

App Name: Şifreli Hoca

Number of Download: 47.000+

Google Play Point: 4,7

Horizontal Symmetry Value: 0.10

Vertical Symmetry Value: 0.02

Total Symmetry Value: 0.06

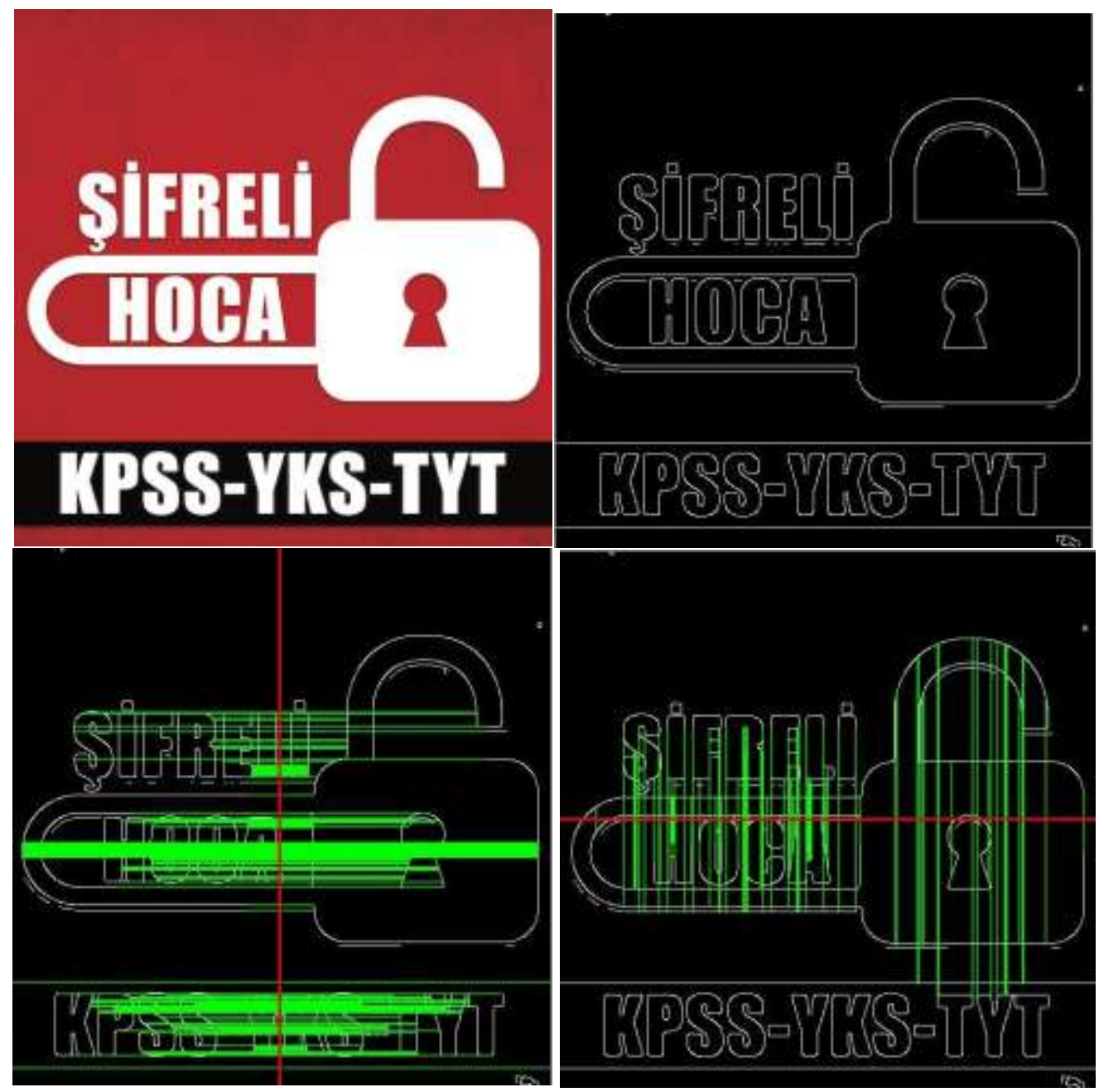

Fig 20. Image Processing Results of Application 9

In Figure 20 image processing steps of the application 9 icon are shown. Image processing steps of application 9 icon are shown in Picture 19. Sparse density was observed in the number of matching white pixels in the horizontal plane and the horizontal symmetry value was determined as 0.10 . Sparse density was observed in the number of matching white pixels on the vertical axis and the vertical symmetry value was determined as 0.02 . The total symmetry value was determined as 0.06 . 


\subsection{Results of Application Number 10}

App Name: KPSS Genel Kültür Yarışması 2018

Number of Download: 10.000+

Google Play Point: 4,2

Horizontal Symmetry Value: 0.20

Vertical Symmetry Value: 0.12

Total Symmetry Value: 0.16

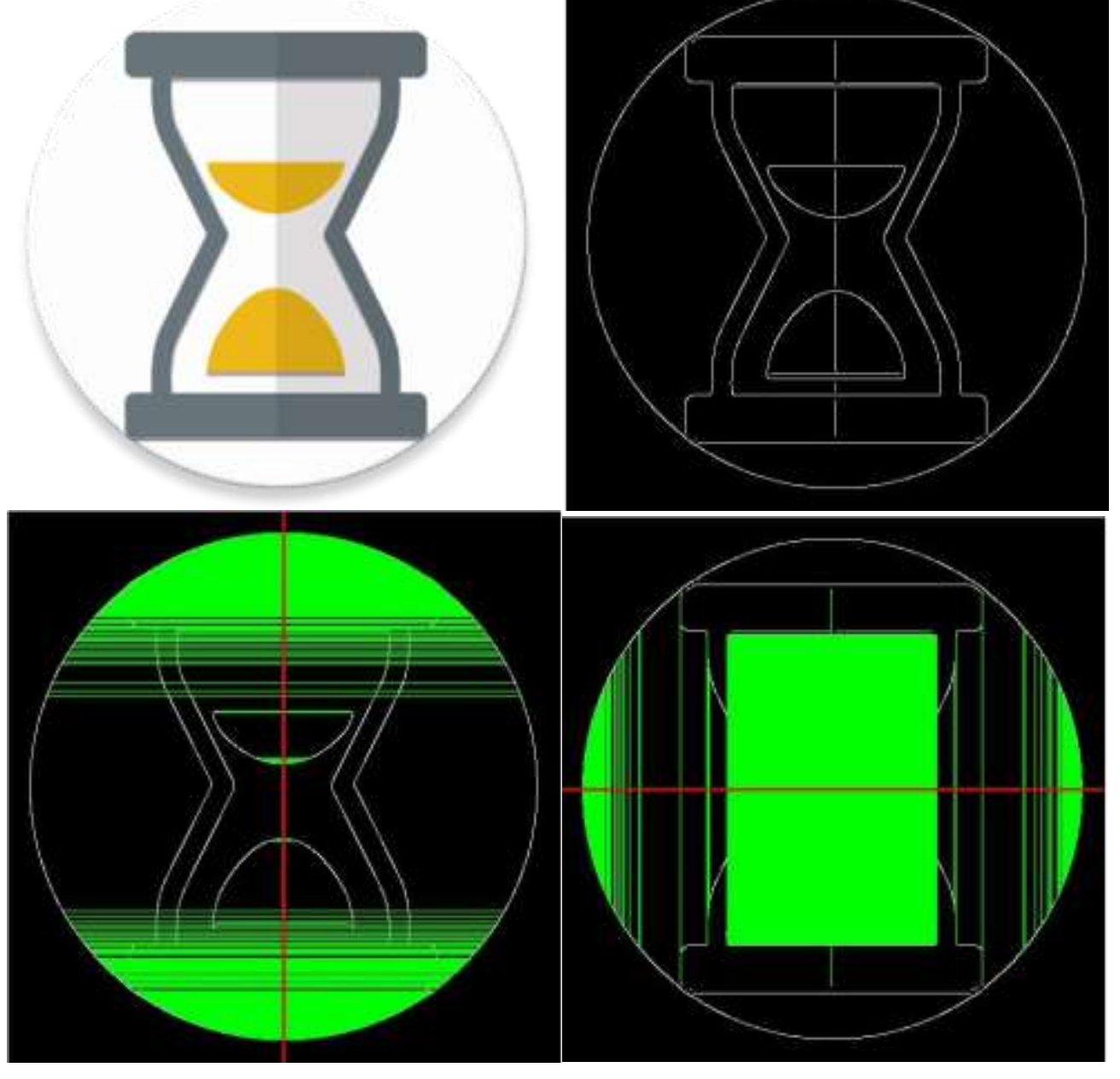

Fig 21. Image Processing Results of Application 10

In Figure 21 image processing steps of the application 10 icon are shown. Excessive density was observed in the number of matching white pixels in the horizontal plane and the horizontal symmetry value was determined as 0.20. Excessive density was also observed in the number of matching white pixels on the vertical axis and the vertical symmetry value was determined as 0.12 . The total symmetry value was determined as 0.16 . 
Table 1. Matlab and Survey Values of App Icons

\begin{tabular}{|c|c|c|}
\hline ICON & MATLAB VALUE & SURVEY VALUE \\
\hline 1 & 0,09 & 2,96 \\
\hline 2 & 0,12 & 3,37 \\
\hline 3 & 0,03 & 3,05 \\
\hline 4 & 0,04 & 3,24 \\
\hline 5 & 0,03 & 2,52 \\
\hline
\end{tabular}


6

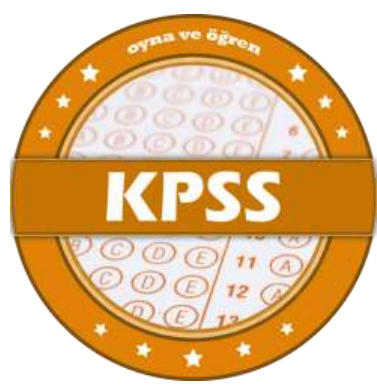

7

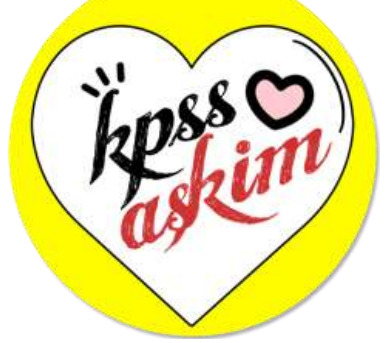

8

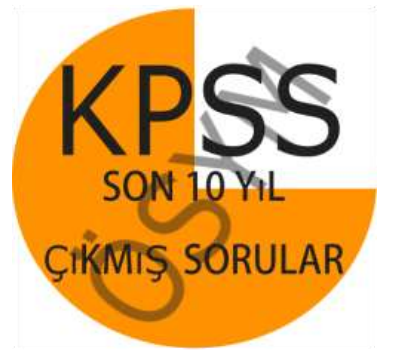

9

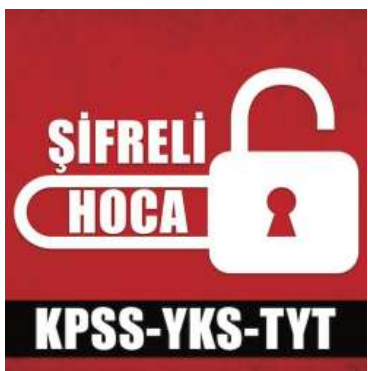

10

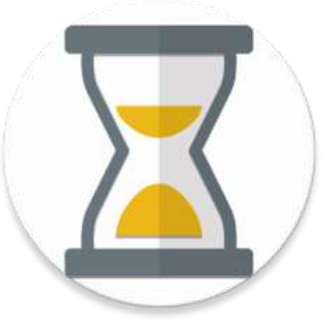

0,17

3,22

0,04

2,23

0,07

3,15

0,06

3,16

0,16

2,77

In Table 1 avarage points of icons from survey added to the matlab points of icons. 


\subsection{Results of Binary Logistic Regression}

Table 2. Result of Confusion Matrix

\begin{tabular}{|c|c|c|c|}
\hline & $\begin{array}{l}\text { AESTHETIC }(>= \\
2.9)\end{array}$ & $\begin{array}{l}\text { NOT AESTHETIC } \\
(>=2.9)\end{array}$ & \\
\hline$(>=0.08)$ & 3 & 1 & $4(\% 75)$ \\
\hline \multirow[t]{2}{*}{$(<0.08)$} & 4 & 2 & $6(\% 33)$ \\
\hline & 7 & 3 & $(\% 50)$ \\
\hline
\end{tabular}

Table 2 shows the grouping of 10 icons according to their data and limit values. From the collected data, the general average of Matlab data was found to be 0.08 and accepted as the grouping limit value for Matlab values. The general average of the survey values was found to be 2.9 and accepted as limit value for grouping.

In the $2 \times 2$ error matrix created for the binary logistic regression analysis, there were 3 icons with both matlab value and survey value exceeding the average, and 1 icon with matlab value exceeding the average but the survey value not exceeding the average. There were 2 icons with both the matlab value and the survey value not exceeding the average, and there were 4 icons whose matlab value could not exceed the average, but the survey value exceeded the average.

\section{Discussion}

According to the data obtained from the error matrix, the sensitivity of our prediction model for positive cases is $75 \%$. Our model showed high precision in predicting positive results. However, for negative situations, the sensitivity rate of our model remained at 33\%. Our model showed low sensitivity in compensating for negative results. The total sensitivity rate of our model was found to be $50 \%$.

It has been observed that the icons whose symmetry values we measure on the computer exceed the general average also exceed the general average value at a high rate in the survey values, but in the opposite case, the same high rate does not appear for the icons below the average. It has been observed that some icons whose symmetry values are below average can also be liked by the respondents. The thesis that "the more symmetry value increases, the more aesthetic taste increases", which we created by taking the road from previous similar studies, was partially correct. 


\section{Conclusions}

In addition to many previous image processing techniques proposed for symmetry ratio determination, a new model is proposed in this study by revealing the white pixels of the picture with the "canny" method, one of the features of the matlab program, and calculating the equidistant ones around the axes. More comprehensive models can be created by adapting the models and codes we proposed to languages with programs such as R, Python and libraries for image processing such as the OpenCv.

In addition to the SIFT Detector method used by Cornelius and Loy (2006) [8], the numerical values of the symmetry can be extracted as well as the symmetry determination in 3D pictures by adding Matlab codes we used.

Numerical values can also be deduced in addition to the symmetry determination with adding our matlab codes to symmetry study which is proposed by Shi et al. (2018) [12], made over a line passing through the center of gravity of the shape formed based on transferring the contours of the picture to the $x$ and $y$ plane in the Matlab program.

When the Ribbon Detection method proposed by Widynski et al. (2014) [14] is similar to the "canny" method we use to draw the outline of a picture which is used to extract the linear lines of the objects in the picture to find regional symmetries combined with the method we proposed, a useful model might be created for the calculation of individual symmetry values of objects in different parts of the picture.

In order to guide the future studies on aesthetics and symmetry, an aesthetic detection model is proposed in this study. These findings, which are found only for mobile application icons, show us that the symmetry in designs such as book cover, video cover photos (thumbnail), commercial company logo, product packaging etc. can create an aesthetic perception and contribute positively to marketing. 


\section{References}

1. Berge, Z. L., \& Muilenburg, L. (Eds.) (2013). Handbook of mobile learning. (p.3-4). New York, NY: Routledge

2. Islam, R., \& Mazumder, T. (2010). Mobile application and its global impact, International Journal of Engineering \& Technology (IJEST), 10(6), 72-78. Erişim adresi: https://www.researchgate.net/profile/Dr_Md_Rashedul_Islam/publication/3080222 97_Mobile_application_and_its_global_impact/links/5991fbafa6fdcc53b79b606d/M obile-application-and-its-global-impact.pdf

3. Cuello, J., \& Vittone, J. (2013). Designing mobile apps. California, SV: Createspace

4. Peirce, C. S. (1932). In C. Hartshorne \& P. Weiss, Eds. The Collected Papers of Charles Sanders Peirce, Vol. 2. Cambridge, MA: Harvard University Press. (Excerpted in J. Buchler, ed. Philosophical Writings of Peirce. New York: Dover, 1955.)

5. "Effects of the aesthetic design of icons on app downloads: evidence from an android market" by M. Wang and X. Kohne, 2016, Electronic Commerce Research, 17(1), p.88. Copyright 2016 by Springer Science+Business Media New York

6. Lavie, T., \& Tractinsky, N., (2004). Assessing dimensions of perceived visual aesthetics of websites. International Journal of Human-Computer Studies, 60(3), 269-298. https://doi.org/10.1016/j.ijhcs.2003.09.002

7. Moshagen, M., \& Thielsch, M. T. (2010). Facets of visual aesthetics. International Journal of Human-Computer Studies, 68(10), 689-709. https://doi.org/10.1016/j.ijhcs.2010.05.006

8. Cornelius, H., \& Loy, G. (2006, Haziran). Detecting bilateral symmetry in perspective. 2006 Conference on Computer Vision and Pattern Recognition Workshop, (s. 191) içinde. Piscataway, NJ: IEEE. https://doi.org/10.1109/CVPRW.2006.63

9. Dakin, S. C., \& Herbert, A. M. (1998). The spatial region of integration for visual symmetry detection. Proc of the Royal Society London B. Biological Science, 265, 659664. https://doi.org/10.1098/rspb.1998.0344

10. Hauagge, D. C., \& Snavely, N. (2012, Haziran). Image matching using local symmetry features. Computer Vision and Pattern Recognition (CVPR), 2012 IEEE Conference on IEEE (s. 206-213) içinde. New York, NY: ACM Press. Erişim Adresi: https://www.cs.cornell.edu/ snavely/publications/papers/hauagge_symdesc_cvpr2 012.pdf

11. Locher, P. J., \& Nodine, C. F. (1985, Eylül). Symmetry catches the eye, Third European Conference on Eye Movements, içinde (s. 353-361). North Holland: Elsevier. https://doi.org/10.1016/B978-0-444-70113-8.50051-5

12. Shi, P., Zheng, X. , Ratkowsky, D. A., Li, Y., Wang, P., \& Cheng, L. A. (2018). Simple Method for Measuring the Bilateral Symmetry of Leaves. Symmetry 2018, 10, 118. https://doi.org/10.3390/sym10040118

13. Wang, M., \& Li, X. (2017). Effects of the aesthetic design of icons on app downloads: evidence from an android market. Electronic Commerce Research, 17(1), 83-102. https://doi.org/10.1007/s10660-016-9245-4

14. Widynski, N., Moevus, A., \& Mignotte, M. (2014). Local symmetry detection in natural images using a particle filtering approach. IEEE Transactions on Image Processing, 23(12), 5309-5322. https://doi.org/10.1109/TIP.2014.2365140 
15. Chertok, M., \& Keller, Y. (2010). Spectral symmetry analysis. IEEE Transactions on Pattern Analysis and Machine Intelligence, 32(7), 1227-1238. https://doi.org/10.1109/TPAMI.2009.121 